\title{
Mg line formation in late-type stellar atmospheres
}

\section{Calculations in a grid of 1D models ${ }^{\star}$}

\author{
Y. Osorio and P. S. Barklem \\ Theoretical Astrophysics, Department of Physics and Astronomy, Uppsala University, Box 516, 75120 Uppsala, Sweden \\ e-mail: yeisson.osorio@physics.uu.se
}

Received 13 July 2015 / Accepted 13 October 2015

\section{ABSTRACT}

\begin{abstract}
Context. $\mathrm{Mg}$ is the $\alpha$ element of choice for Galactic population and chemical evolution studies because it is easily detectable in all late-type stars. Such studies require precise elemental abundances, and thus departures from local thermodynamic equilibrium (LTE) need to be accounted for.

Aims. Our goal is to provide reliable departure coefficients and equivalent widths in non-LTE, and for reference in LTE, for diagnostic lines of Mg studied in late-type stars. These can be used, for example, to correct LTE spectra and abundances.

Methods. Using the model atom built and tested in the preceding paper in this series, we performed non-LTE radiative transfer calculations in a grid of 3945 stellar 1D atmospheric models. We used a sub-grid of 86 models to explore the propagation of errors in the recent atomic collision calculations to the radiative transfer results.

Results. We obtained departure coefficients for all the levels and equivalent widths (in LTE and non-LTE) for all the radiative transitions included in the "final" model atom presented in Paper I. Here we present and describe our results and show some examples of applications of the data. The errors that result from uncertainties in the collisional data are investigated and tabulated. The results for equivalent widths and departure coefficients are made freely available.

Conclusions. Giants tend to have negative abundance corrections while dwarfs have positive, though small, corrections. Error analysis results show that uncertainties related to the atomic collision data are typically on the order of 0.01 dex or less, although for few stellar models in specific lines uncertainties can be as large as 0.03 dex. As these errors are less than or on the same order as typical corrections, we expect that we can use these results to extract $\mathrm{Mg}$ abundances from high-quality spectra more reliably than from classical LTE analysis.
\end{abstract}

Key words. line: formation - stars: abundances

\section{Introduction}

Magnesium plays an important role in Galactic population and chemical evolution studies. It is released during type II supernovae occurring at the end of the life of massive stars, and so tracing the evolution of $\mathrm{Mg}$ is an important tool for our understanding of the evolution of the Galaxy. Mg lines are observed in all late-type stars, even in very metal-poor stars (e.g. Christlieb et al. 2002; Frebel et al. 2005) and in a wide spectral range from the UV to the IR.

There have been a number of previous non-local thermodynamic equilibrium (non-LTE) studies of $\mathrm{Mg}$ in different types of stars (e.g. Athay \& House 1962; Shimanskaya et al. 2000; Przybilla et al. 2001; Langangen \& Carlsson 2009; Mashonkina 2013), with particular interest in the MgI emission lines observed in the Sun and some giant stars (Mauas et al. 1988; Chang et al. 1991; Carlsson et al. 1992; Uitenbroek \& Noyes 1996; Sundqvist et al. 2008). The main conclusions from this collection of studies is the increase of non-LTE effects with decreasing metallicity $([\mathrm{Fe} / \mathrm{H}])$ and the demonstration that the IR Mg emission lines form because of non-LTE effects and have a photospheric origin. In the first paper of this series (Osorio et al. 2015, hereafter Paper I), we constructed a new Mg model atom for

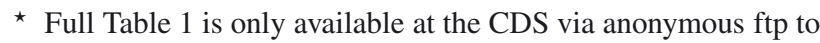
cdsarc.u-strasbg.fr (130.79.128.5) or via

http://cdsarc.u-strasbg.fr/viz-bin/qcat?J/A+A/586/A120

The same data is accessible via the INSPECT project http://

inspect. coolstars19. com
non-LTE studies in late-type stellar atmospheres; new quantum mechanical calculations were performed and implemented in the model atom. Comparison of the non-LTE line profiles with observed spectra and an investigation of the effect of different collisional processes in four stellar atmospheric models was also performed in Paper I.

In this paper, we present a grid of non-LTE calculations for $\mathrm{Mg}$ in late-type stars, based on this model atom. Such largescale calculations in grids of atmospheric models have been performed for a number of elements, e.g. lithium (Carlsson et al. 1994; Lind et al. 2009, using 72 and 392 atmospheric models respectively), carbon (Fabbian et al. 2006, 176 models), sodium (Lind et al. 2011, 764 model atmospheres), and iron (Lind et al. 2012, 2900 models). A grid for Mg has been calculated recently by Merle et al. (2011) using 450 models for $\mathrm{Mg}$ lines with a model atom that neglects collisions with $\mathrm{H}$ and uses electron collisional data from quantum mechanical calculations (when available), though the sources are not specified.

For this work, we use the MARCS ${ }^{1}$ grid of stellar atmospheres (Gustafsson et al. 2008) to perform non-LTE calculations of $\mathrm{Mg}$ using the final model atom, called " $\mathrm{F}$ " in Paper I, and present non-LTE, and for reference LTE, equivalent widths. From the equivalent widths, corresponding abundance corrections can be calculated for a set of abundances in each atmosphere.

1 http://marcs.astro.uu.se/ 
This paper is structured as follows. In Sect. 2 we briefly describe the model atom, the atmospheric models used, and the non-LTE spectrum calculations; the details are in Paper I. In Sect. 3 we present the results and analyse the abundance corrections on the stellar grid (Sect. 3.2), study the propagation of uncertainties that result from uncertainties in the collisional data used (Sect. 3.3), and show examples of possible applications of our results (Sect. 3.4). Finally in Sect. 4, we present our conclusions.

\section{NLTE modelling}

Here we recap the most important aspects of the model atom, methods, and codes used for solving the non-LTE radiative transfer problem. We then describe the application to the large-scale calculations performed in this work.

For solving the restricted non-LTE radiative transfer (RT) problem we used the MULTI code (Carlsson 1986, 1992), which uses the plane-parallel geometry setup and treats a given atomic species, here $\mathrm{Mg}$, in the trace element approximation. In this work, for the calculation of $\mathrm{Mg}$ non-LTE line formation, we use the so called "final" model atom built and tested in Paper I, Model F. This atom has 143 levels (108 for Mg I 34 for Mg II and the ground state of $\mathrm{Mg}$ III) and 1185 lines. Energy levels and $f$ values were taken mostly from the NIST database (Ralchenko et al. 2010). Stark broadening data was taken mostly from Dimitrijevic \& Sahal-Brechot (1996) and van der Waals broadening from the ABO theory (Barklem et al. 1998) and new calculations also presented in Paper I. New electron collisional data was calculated in Paper I and used in the F model atom, while for transitions not covered by the new calculations, we used the impact parameter (IP) method (Seaton 1962). Hydrogen collisional data between low-lying states was taken from Barklem et al. (2012) and the method of Kaulakys (1986) used for remaining transitions. Additionally, some variations of this model atom were created for testing the sensitivity of the results to the new collisional data from Barklem et al. (2012) and that from Paper I (see Sect. 3.3). For more details about the model atom see Paper I.

We performed non-LTE calculations for $\mathrm{Mg}$ in 3945 MARCS stellar atmospheric models with ranges of effective temperature $T_{\text {eff }}=[2500,8000] \mathrm{K}$, surface gravity $\log g=[-0.5,5.0]\left(\mathrm{cm} / \mathrm{s}^{2}\right)$, and metallicity $[\mathrm{Fe} / \mathrm{H}]=[-5.0,1.0]$ dex. For each stellar model we used depth-independent micro-turbulent velocities of $\xi=1.0$ and $2.0 \mathrm{~km} \mathrm{~s}^{-1}$ and, additionally, $\xi=3.0 \mathrm{~km} \mathrm{~s}^{-1}$ for the stellar models with $\log g \leq 3.5\left(\mathrm{~cm} / \mathrm{s}^{2}\right)$. Approximately $70 \%$ of the MARCS atmospheric models used in this work are spherical model atmospheres, the remaining $30 \%$ are plane-parallel models used for dwarfs, where the high surface gravity means the atmosphere is sufficiently thin for this approximation to be valid. The use of spherical atmospheric models in plane-parallel RT solvers was studied by Heiter \& Eriksson (2006). They recommend the use of spherical atmospheric models in planeparallel RT solvers when spherical atmospheric models, but not spherical RT solvers, are available. However, they point out that for low $T_{\text {eff }}$ and $\log g$ (cool giant) models, a plane-parallel atmosphere/RT solver combination provides results that are closer to the spherical model atmosphere/RT solver combination than the hybrid spherical model atmosphere/plane-parallel RT solver (see Heiter \& Eriksson 2006 for details).

For each stellar model, we calculated LTE and non-LTE equivalent widths ( $w^{*}$ and $w$ respectively) for 23 abundance points between $A(\mathrm{Mg})=2.6$ and $8.6 \mathrm{dex}^{2}$. This allows abundance corrections to be deduced for a wide range of possible values of $[\mathrm{Mg} / \mathrm{Fe}]$. A subset of 86 stars was also used for studying how the uncertainties in the recent collisional calculations propagate to the final non-LTE equivalent widths and abundance corrections.

In the calculations, we made use of the two options for starting procedures in MULTI, which were found to lead to the most reliable convergence. For low gravity models LTE populations are used as a starting point. For high $\log g$ stellar models, convergence was attained more efficiently with starting populations that correspond to the solution of the statistical equilibrium equations with zero radiation field.

\section{Results}

We calculated departure coefficients $b_{i}$ of the 143 levels of the "F" model atom in Paper I. We also calculated equivalent widths in LTE and non-LTE for all the 1185 radiative transitions in the "F" model atom, but focus on a subset of 19 lines for which analysis is performed. The behaviour of $\mathrm{Mg}$ abundance corrections, $\triangle A(\mathrm{Mg})$, defined as

$\Delta A(\mathrm{Mg})=A(\mathrm{Mg})_{\mathrm{NLTE}}-A(\mathrm{Mg})_{\mathrm{LTE}}$,

varies across the stellar grid, with magnesium abundance, and with the line under study.

\subsection{Departure coefficients}

For each stellar model and $\mathrm{Mg}$ abundance calculated, we obtained departure coefficients ${ }^{3} b_{i}$ as a function of optical depth at $500 \mathrm{~nm}\left(\tau_{500 \mathrm{~nm}}\right)$ of the 143 levels used in the " $F$ " model atom from Paper I. The departure coefficients can be used in radiative transfer (RT) codes to include non-LTE effects in spectral fitting codes without the need to solve the non-LTE RT problem, e.g. the SME code (Valenti \& Piskunov 1996, and updates) can use $b_{i}$ 's in this way (as demonstrated in Sect. 4.1 of Paper I). The behaviour of $b_{i}$ with $\mathrm{A}(\mathrm{Mg})$ is smooth, yet not simple, especially for the low-lying excited levels of $\mathrm{Mg} \mathrm{I}$; see for example Fig. 1, which shows how $b_{i}$ for the first excited level of $\mathrm{Mg}$ I varies with $\mathrm{Mg}$ abundance and $\tau_{500 \mathrm{~nm}}$ for two stars differing only in metallicity. Thus, results must be tabulated and to obtain reliable results in possible interpolation, the behaviour of $b_{i}$ with $\mathrm{A}(\mathrm{Mg})$ must be sufficiently sampled. Each of the 3945 MARCS model atmospheres has 56 depth points (distributed between values of the Rosseland mean optical depth $\tau_{\text {Ross }}=10^{-5}$ and $\tau_{\text {Ross }}=100$, see Gustafsson et al. 2008 for details) for which we obtained $\mathrm{Mg}$ populations for each of the 143 levels in the model atom (together with other data), and we calculated $23 \mathrm{Mg}$ abundance values. The resulting departure coefficients are also available at CDS.

\subsection{Equivalent widths and abundance corrections}

We present a set of non-LTE, and for reference LTE, equivalent widths for 19 diagnostic lines of $\mathrm{Mg}$ commonly observed in late-type stellar spectra. Abundances range from $A(\mathrm{Mg})=2.6$ to 8.6 dex sampled with 23 values for each of the 3945 1D stellar atmospheric models used from the MARCS grid. Table 1

\footnotetext{
2 We use usual notation for element abundances $A(\mathrm{X})=\log _{10} \frac{n_{\mathrm{X}}}{n_{\mathrm{H}}}+12$, where $n_{x}$ is the number density of species $\mathrm{X}$.

3 Defined here as the ratio between non-LTE and LTE populations for level $i, b_{i}=n_{i} / n_{i}^{*}$.
} 

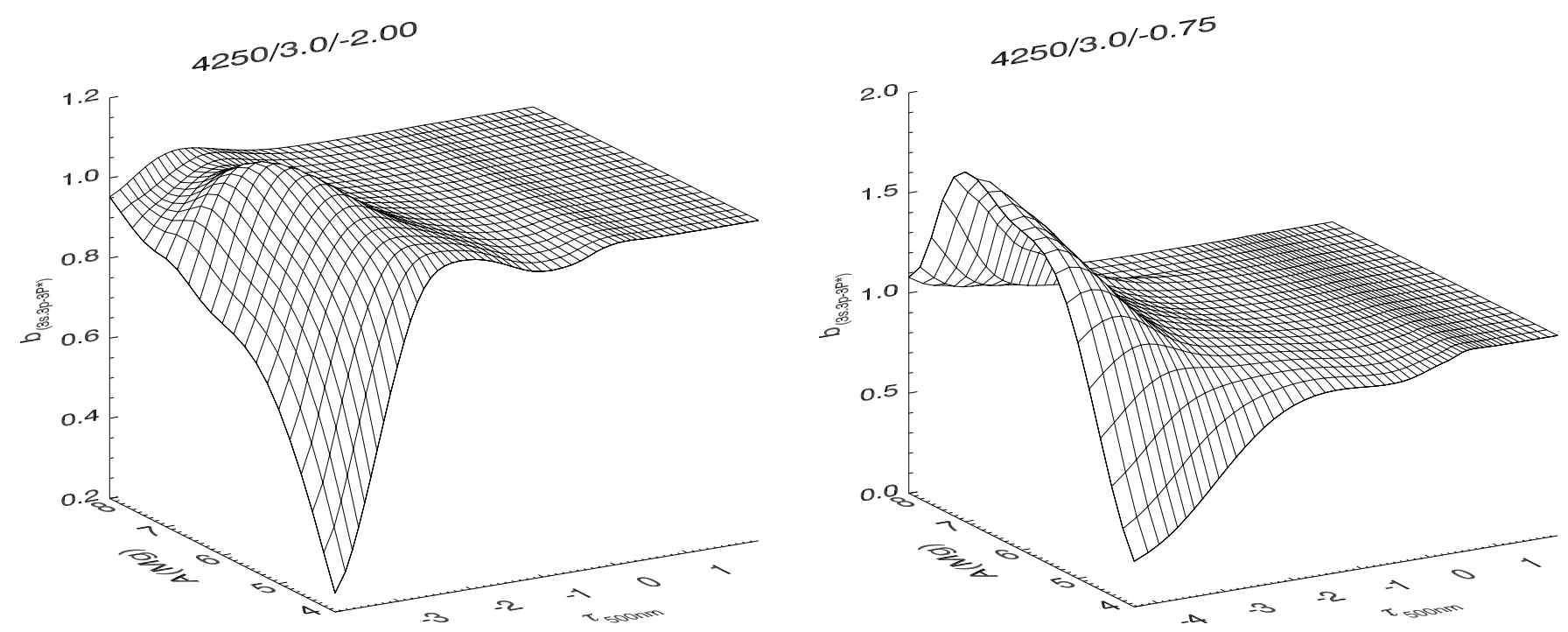

Fig. 1. Departure coefficient $b$ of the first excited level of Mg I in stellar models with $T_{\text {eff }} / \log g /=4250 / 3.0$ and $[\mathrm{Fe} / \mathrm{H}]=-2.0(l e f t)$ and -0.75 (right) as function of optical depth (at $500 \mathrm{~nm}$ ) and $\mathrm{Mg}$ abundance.

shows the format of the file available at CDS. The first four columns show parameters of the stellar model $T_{\text {eff }}, \log g,[\mathrm{Fe} / \mathrm{H}]$, and micro-turbulent velocity $\xi$, the fifth column is the abundance of $\mathrm{Mg}, A(\mathrm{Mg})$, used, and the remaining columns report the predicted LTE equivalent widths $w^{*}$ and non-LTE equivalent widths $w$ for each line $\lambda$, in the dimensionless form $\log _{10}(w / \lambda)$.

We selected 19 lines of $\mathrm{MgI}$, some of them presented in Table 1 of Paper $\mathrm{I}^{4}$, those lines are commonly used for $\mathrm{Mg}$ studies of late-type stars. For example the $\mathrm{Mg}$ I b lines, which we included, are observed in a wide range of stellar parameters, even in very metal-poor stars. The inter-combination line at $4571 \AA$ and the $4703 \AA$ line, used commonly for Mg abundance determinations, are also included. Among the studied transitions there are the $\mathrm{Mg}$ lines covered by the GALAH (5711 and $7691 \AA$ ) and Gaia-ESO (5528 and $8806 \AA$ ) surveys. For the purposes of our discussion, we focus on these last four lines as representative of the typical behaviour of the abundance corrections $\triangle A(\mathrm{Mg})$, defined here as the difference between the abundance in non-LTE minus the abundance in LTE corresponding to the same $w$ for the given $\mathrm{Mg}$ line. The abundance corrections derived from our equivalent width results for these lines are shown in Fig. 2.

The physical conditions in the atmosphere and the interaction of $\mathrm{Mg}$ with electrons, hydrogen atoms and photons determines the behaviour of the Mg populations, spectral lines and thus abundance corrections, which can be described for different regions of the stellar parameter space (see Fig. 2). For cool giant atmospheric models, corrections tend to be negative and the most extreme values are around $[\mathrm{Fe} / \mathrm{H}] \sim-2$ for the 5528 and $8806 \AA$ lines, while the $7691 \AA$ line in cool giants has stronger corrections with increasing metallicities. F and G dwarf models have for most lines small positive corrections at $[\mathrm{Fe} / \mathrm{H}] \gtrsim-3$ that increase at lower metallicities, in particular for the $7691 \AA$ line. The higher density in dwarfs tends to thermalise the populations and therefore the corrections weaken towards higher $\log g$. The transition from negative to positive corrections is more dependent on $T_{\text {eff }}$ than on $\log g$ or $[\mathrm{Fe} / \mathrm{H}]$.

\footnotetext{
4 On request, we can provide similar data for any of the 1185 lines in the model atom described in Paper I.
}

The effect of micro-turbulence $\xi$ in the non-LTE abundance corrections is expected to be small given that obtaining abundance corrections is a differential procedure with respect to equivalent widths $w$. The strongest dependence of the obtained corrections with $\xi$ is of the order of 0.02 dex and occurs when the line is almost saturated. Weak lines are formed in the same region of the atmosphere so non-LTE corrections behave similar for different values of $\xi$. For medium strength lines, not yet saturated in the line core, micro-turbulence affects the entire profile but the core and wings of the line are formed at different depths where different non-LTE mechanisms may dominate (Mashonkina 2010) causing the LTE and non-LTE profiles to behave slightly differently with respect to each other at different $\xi$. Once the line saturates, the wings start to be independent of $\xi$ and the non-LTE effects start to depend again only on the same depth in the atmosphere (where the core is formed). Abundance corrections become less dependent on $\xi$ with increasing abundance for saturated lines. Below we describe the behaviour of $\Delta A(\mathrm{Mg})$ for different lines in the stellar parameter space $\left\{T_{\text {eff }}\right.$, $\log g,[\mathrm{Fe} / \mathrm{H}]\}$.

At $T_{\text {eff }} \sim 5000 \mathrm{~K}$ the populations of $\mathrm{Mg}$ I and $\mathrm{Mg}$ II are comparable and departure coefficients tend to be close to unity $\left(b_{i} \sim 1\right) . \mathrm{Mg} \mathrm{I}$ is therefore not a minority species at lower $T_{\text {eff }}$; $\mathrm{Mg}$ Ipopulation is larger than that of $\mathrm{Mg}$ II in line formation regions. At $T_{\text {eff }} \lesssim 3000 \mathrm{~K}$ the $\mathrm{Mg}$ I ground level, $3 \mathrm{~s}^{1} \mathrm{~S}$, and its first two excited states, $3 \mathrm{p}^{3,1} \mathrm{P}$, are thermalised at $\tau_{500 \mathrm{~nm}} \geq-1$. The lower state of the 5711 and $5528 \AA$ lines is $3 \mathrm{p}^{1} \mathrm{P}$, which is strongly collisionally coupled with the ground level of $\mathrm{Mg}$ I. At $T_{\text {eff }} \sim 4500 \mathrm{~K}$, when the ground level of $\mathrm{Mg}$ II starts to thermalise, the Mg I low-lying levels are still close to LTE populations leading to almost LTE conditions in all the lines formed in atmospheres around these values of $T_{\text {eff. }}$. There is a less clear dependency of the behaviour of $b_{i}$ 's with $\log g$ and metallicity, though at higher $\log g$ the range in $T_{\text {eff }}$ where the atomic levels are close to LTE conditions broadens (see Fig. 2).

By checking the $b_{i}$ we can study the interplay of the different population and de-population mechanisms that produce the final line profiles and $w$ 's. At $T_{\text {eff }} \gtrsim 5000 \mathrm{~K} \mathrm{Mg}$ I starts to become a minority species and the ground level of $\mathrm{Mg}$ II is thermalised leading to underpopulated $\mathrm{Mg}$ I levels with respect 
A\&A 586, A120 (2016)

Table 1. Segment of the file containing the LTE and non-LTE equivalent width results.

\begin{tabular}{|c|c|c|c|c|c|c|c|c|c|c|c|c|c|c|c|}
\hline \multicolumn{16}{|c|}{ * values in LTE and NLTE are $\log (\mathrm{w} / \mathrm{lambda})$. lambda is in Angstroms } \\
\hline \multirow[t]{2}{*}{$* T_{\text {eff }}$} & \multirow{2}{*}{$\log (g)$} & \multirow{2}{*}[\mathrm{Fe}/\mathrm{H}]{} & \multirow{2}{*}{$v_{\text {turb }}$} & \multirow{2}{*}{$A(\mathrm{mg})$} & \multicolumn{2}{|c|}{3832.302} & \multirow{2}{*}{$\frac{\ldots}{\ldots}$} & \multicolumn{2}{|c|}{4571.095} & \multirow{2}{*}{$\frac{\ldots}{\ldots}$} & \multicolumn{2}{|c|}{8806.751} & \multirow{2}{*}{$\ldots$} & \multicolumn{2}{|c|}{21060.951} \\
\hline & & & & & lte & nlte & & lte & nlte & & lte & nlte & & lte & nlte \\
\hline 2500 & 0.00 & 0.50 & 1.00 & 2.60 & -4.7924 & -4.7737 & $\ldots$ & -5.2677 & -5.3026 & $\ldots$ & -7.2368 & -7.1734 & $\ldots$ & -8.6819 & -8.6889 \\
\hline 2500 & 0.00 & 0.50 & 1.00 & 3.00 & -4.7067 & -4.6879 & $\ldots$ & -5.0257 & -5.0540 & $\ldots$ & -6.8477 & -6.7835 & $\ldots$ & -8.2821 & -8.2972 \\
\hline 2500 & 0.00 & 0.50 & 1.00 & 3.60 & -4.6042 & -4.5880 & $\ldots$ & -4.8065 & -4.8230 & $\ldots$ & -6.2937 & -6.2245 & $\ldots$ & -7.6827 & -7.7025 \\
\hline 2500 & 0.00 & 0.50 & 1.00 & 4.00 & -4.5328 & -4.5165 & $\ldots$ & -4.7189 & -4.7298 & $\ldots$ & -5.9634 & -5.8844 & $\ldots$ & -7.2839 & -7.3029 \\
\hline 2500 & 0.00 & 0.50 & 1.00 & 4.60 & -4.3910 & -4.3701 & $\ldots$ & -4.6290 & -4.6349 & $\ldots$ & -5.5557 & -5.4554 & $\ldots$ & -6.6901 & -6.7017 \\
\hline 2500 & 0.00 & 0.50 & 1.00 & 5.00 & -4.2618 & -4.2372 & $\ldots$ & -4.5845 & -4.5885 & $\ldots$ & -5.3450 & -5.2310 & $\ldots$ & -6.3022 & -6.3047 \\
\hline 2500 & 0.00 & 0.50 & 1.00 & 5.30 & -4.1466 & -4.1208 & $\ldots$ & -4.5558 & -4.5588 & $\ldots$ & -5.2140 & -5.0926 & $\ldots$ & -6.0214 & -6.0160 \\
\hline$\ldots$ & $\ldots$ & $\ldots$ & $\ldots$ & & $\ldots$ & $\ldots$ & $\ldots$ & $\ldots$ & $\ldots$ & $\ldots$ & $\ldots$ & $\ldots$ & $\ldots$ & & \\
\hline 4500 & 4.50 & 0.00 & 2.00 & 7.60 & -2.7603 & -2.7606 & $\ldots$ & -4.1783 & -4.1790 & $\ldots$ & -3.9022 & -3.8962 & $\ldots$ & -4.3822 & -4.3810 \\
\hline 4500 & 4.50 & 0.00 & 2.00 & 7.80 & -2.7069 & -2.7072 & $\ldots$ & -4.1113 & -4.1117 & $\cdots$ & -3.8295 & -3.8242 & $\ldots$ & -4.2726 & -4.2708 \\
\hline 4500 & 4.50 & 0.00 & 2.00 & 8.00 & -2.6650 & -2.6652 & $\ldots$ & -4.0398 & -4.0400 & $\ldots$ & -3.7624 & -3.7577 & $\ldots$ & -4.1852 & -4.1829 \\
\hline 4500 & 4.50 & 0.00 & 2.00 & 8.10 & -2.6484 & -2.6486 & $\ldots$ & -4.0031 & -4.0033 & $\ldots$ & -3.7314 & -3.7270 & $\ldots$ & -4.1490 & -4.1465 \\
\hline 4500 & 4.50 & 0.00 & 2.00 & 8.30 & -2.6233 & -2.6235 & $\ldots$ & -3.9297 & -3.9298 & $\ldots$ & -3.6749 & -3.6709 & $\ldots$ & -4.0892 & -4.0863 \\
\hline 4500 & 4.50 & 0.00 & 2.00 & 8.50 & -2.6070 & -2.6071 & $\ldots$ & -3.8583 & -3.8584 & $\ldots$ & -3.6263 & -3.6228 & $\ldots$ & -4.0429 & -4.0394 \\
\hline 4500 & 4.50 & 0.00 & 2.00 & 8.60 & -2.6013 & -2.6014 & $\ldots$ & -3.8242 & -3.8243 & $\ldots$ & -3.6050 & -3.6017 & $\ldots$ & -4.0236 & -4.0199 \\
\hline 4500 & 4.50 & 0.25 & 1.00 & 2.60 & -4.9143 & -4.9958 & $\ldots$ & -7.3019 & -7.4501 & $\ldots$ & -7.0945 & -7.3881 & $\ldots$ & -9.1885 & -8.9062 \\
\hline 4500 & 4.50 & 0.25 & 1.00 & 3.00 & -4.7183 & -4.7723 & $\ldots$ & -6.9030 & -7.0421 & $\ldots$ & -6.6981 & -6.9274 & $\ldots$ & -8.7885 & -8.5428 \\
\hline 4500 & 4.50 & 0.25 & 1.00 & 3.60 & -4.5119 & -4.5408 & $\ldots$ & -6.3086 & -6.4194 & $\ldots$ & -6.1153 & -6.2646 & $\ldots$ & -8.1885 & -8.0462 \\
\hline
\end{tabular}

Notes. The full table is available at the CDS.

to LTE due to over-ionisation. The $5528 \AA$ line has negative $\triangle A(\mathrm{Mg})$ at low $T_{\text {eff }}$, increasing towards the giant region of the parameter space and lower metallicities due to $b_{j} / b_{i}<1$ which become $>1$ at high $T_{\text {eff }}$, with $j>i$ (the location of the transition temperature changes with metallicity and surface gravity). The $5711 \AA$ line has a similar behaviour though not as strong. Other $\mathrm{Mg}$ lines have different behaviour. The Mg I inter-combination line at $4571 \AA$ has only positive $\Delta A(\mathrm{Mg})$ that increase towards high metallicities, due to the UV over-ionisation depopulation of the $\mathrm{Mg}$ I ground level in comparison with LTE $\left(b_{3 \mathrm{~s}^{1} \mathrm{~S}} \lesssim 1\right)$. The $\mathrm{Mg}$ I b lines show no significant $\triangle A(\mathrm{Mg})$ at low $T_{\text {eff }}$ but hot dwarfs have negative corrections at $[\mathrm{Fe} / \mathrm{H}] \sim-2$ and in the most extreme metal-poor atmospheres $(-5<[\mathrm{Fe} / \mathrm{H}]<-3)$, corrections become positive at intermediate $T_{\text {eff }}$. The IR lines at $2.1 \mu \mathrm{m}$ show only positive corrections at high metallicities and strong positive corrections start to appear when the lines becomes too weak to have photon losses that compensate for $b_{j} / b_{i}>1$ in the line formation region.

\subsubsection{Comparison with earlier works}

The previous non-LTE study of Mg that is most similar to ours, in terms of the atomic data used, is the work of Mashonkina (2013). In that work, non-LTE corrections for late-type stars using the recent calculations for $\mathrm{H}$ collisions presented in Barklem et al. (2012; which we also used) were compared with those obtained using the Drawin formula. The main differences between our work and that study are, in addition to the use of different non-LTE codes, differences in electron collisional data, treatment of hydrogen collisional excitation rates for high-lying levels, and the use of MAFAGS-ODF (Fuhrmann et al. 1997) model atmosphere including different opacities by Mashonkina. Comparison for some of the models and lines presented in Table 5 in Mashonkina are in Table 2. In general the agreement is reasonable, considering the differences mentioned above.

The study of Merle et al. (2011) uses, like this work, the MULTI code and MARCS model atmospheres. However, hydrogen collisions are not treated in their modelling. Table 3 in this work is an adaptation of their Table 2, comparing with some of our results. The differences vary with spectral line and stellar parameters, but in general, our corrections are smaller (i.e. our results are closer to LTE). Note that for the $[\mathrm{Fe} / \mathrm{H}]=-2$ models the corrections suggested by Merle et al. are $>0.15 \mathrm{dex}$ while the corrections found by us are significantly smaller $(\sim 0.02 \mathrm{dex})$.

\subsection{Sensitivity to new collisional data}

Collisional processes are a major source of uncertainty in nonLTE radiative transfer calculations. In this section we study the sensitivity of the abundance corrections on the atomic data calculated in Paper I for electron collisions and the calculations for hydrogen collisional data presented in Guitou et al. (2012), Belyaev et al. (2012) and Barklem et al. (2012).

In order to check the sensitivity of our results on the new collisional data we built model atoms with 0.5 and 2.0 times the collisional rates calculated for excitation due to collisions with $\mathrm{H}(\mathrm{CH})$ and charge exchange with $\mathrm{H}(\mathrm{CH})$ from Guitou et al. (2012), Belyaev et al. (2012) and Barklem et al. (2012). These multiplication factors represent an estimate of the typical error of those collisional rates, at least for the transitions with the largest rates. Note, errors in transitions with very small rates may well be higher; however, we expect these rates to be less important in the modelling. The same factors $(0.5$ and 2.0$)$ were used to study the sensitivity of the rates due to electron collisions (CE) calculated in Paper I, although the error in the CE collisional rates is expected to be less for transitions between the lowest lying states, typically $\sim 20 \%(<0.1 \mathrm{dex})$. These new model atoms were used in non-LTE calculations on a sub-grid of 86 stellar models with $3500 \leq T_{\text {eff }}[\mathrm{K}] \leq 7500,1 \leq \log g\left[\mathrm{~cm} / \mathrm{s}^{2}\right] \leq 4$ and $-4 \leq[\mathrm{Fe} / \mathrm{H}] \leq 0.5 \mathrm{dex}$.

In general, we note that the interplay of the $\mathrm{Mg}$ I level populations and different excitation/ionisation processes together with the transition from $\mathrm{Mg}$ II to $\mathrm{Mg}$ I as minority species, leads to different effects of the collisional processes on the final nonLTE populations. For example, in Paper I we pointed out that in some cases, adding collisional interactions to the calculations does not necessarily lead to thermalisation.

At very low $T_{\text {eff }}(\$ 3500 \mathrm{~K})$, the ground state of $\mathrm{Mg} \mathrm{I}\left(3 \mathrm{~s}^{2}{ }^{1} \mathrm{~S}\right)$ and the first two excited states $\left(3 \mathrm{p}^{3,1} \mathrm{P}\right)$ are thermalised so changes in the collisional data do not make their populations to depart from LTE in the line formation regions. The $3 p{ }^{1} \mathrm{P}$ state is the lower level of the 5711 and the $5528 \AA$ lines, so abundance corrections of these lines come from the departure coefficients $b_{i}$ of the upper levels $\left(5 \mathrm{~s}{ }^{1} \mathrm{~S}\right.$ for the $5711 \AA$ and $4 \mathrm{~d}^{1} \mathrm{D}$ for the $5528 \AA$ lines). $b_{5 \mathrm{~s}^{1} \mathrm{~S}}$ and $b_{4 \mathrm{~d}^{1} \mathrm{D}}$ are less than unity in the line formation region, which strengthens the non-LTE lines when compared with LTE making the abundance corrections negative. The upper levels of these lines are sensitive mostly to $\mathrm{CH}$, and so are 
Y. Osorio and P. S. Barklem: Mg Line formation in late-type stellar atmospheres

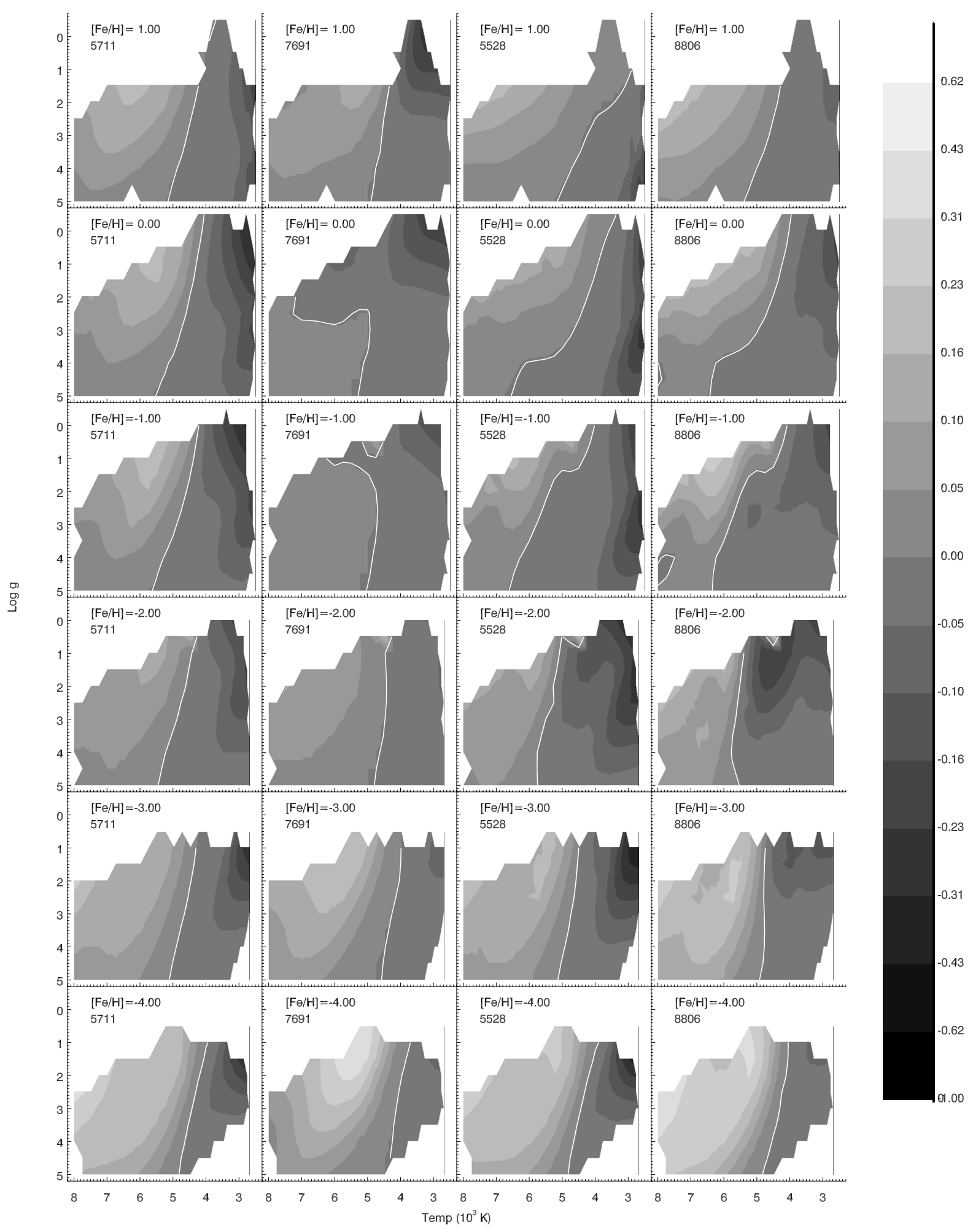

Fig. 2. Abundance corrections at $[\mathrm{Mg} / \mathrm{Fe}]=0$ for $[\mathrm{Fe} / \mathrm{H}] \geq 0$ and at $[\mathrm{Mg} / \mathrm{Fe}]=0.4$ for $[\mathrm{Fe} / \mathrm{H}]<0$ in some of the atmospheric models of the stellar grid used in this work. Micro-turbulent velocity $\xi=2.0 \mathrm{~km} \mathrm{~s}^{-1}$ in all the models in the plot. Each column shows corrections for a different $\mathrm{Mg}$ I line. Note that the colour scaling is not linear. The white line shows the contour of zero abundance correction.

the spectral lines involving them. $\mathrm{CH} 0$ does not affect the level populations in these atmospheres. The $8806 \AA$ line has $3 \mathrm{p}^{1} \mathrm{P}$ as its lower level and $3 \mathrm{~d}^{1} \mathrm{D}$ as its upper level. The $3 \mathrm{~d}^{1} \mathrm{D}$ level is particularly sensitive to $\mathrm{CH}$ in the atmospheric region where the core of the $8806 \AA$ line is formed leading to a $\mathrm{CH}$-sensitive nonLTE equivalent width $w$.
At higher $T_{\text {eff }}(\sim 4000 \mathrm{~K})$, populations become sensitive to $\mathrm{CHO}$ and $\mathrm{CE}$. In these stars the $\mathrm{Mg}$ I level populations are almost thermalised in the line formation region $\left(\tau_{500 \mathrm{~nm}} \sim-0.5\right)$ though slightly underpopulated with respect to LTE; thermalisation is stronger at higher surface gravities. The $\mathrm{CH} 0$ rate coefficients of the levels around $4 \mathrm{~s}{ }^{1} \mathrm{~S}$ make ionising transitions of these levels 
Table 2. Comparison of the $\mathrm{Mg}$ abundance corrections $\Delta A(\mathrm{Mg})$ at $[\mathrm{Mg} / \mathrm{Fe}]=0.4$ dex obtained from us (Oso) with those from Mashonkina (2013, Mas) for selected lines and model atmospheres.

\begin{tabular}{|c|c|c|c|c|c|c|c|c|c|c|c|c|}
\hline \multirow[t]{2}{*}{ Model } & \multicolumn{2}{|c|}{$3829 \AA$} & \multicolumn{2}{|c|}{$5172 \AA$} & \multicolumn{2}{|c|}{$4571 \AA$} & \multicolumn{2}{|c|}{$4703 \AA$} & \multicolumn{2}{|c|}{$5528 \AA$} & \multicolumn{2}{|c|}{$5711 \AA$} \\
\hline & Oso & Mas & Oso & Mas & Oso & Mas & Oso & Mas & Oso & Mas & Oso & Mas \\
\hline $6000 / 4.0 /-1.0$ & 0.02 & 0.05 & 0.00 & 0.04 & 0.03 & 0.08 & 0.01 & 0.01 & -0.03 & -0.03 & 0.03 & 0.04 \\
\hline $6000 / 4.0 /-2.0$ & 0.01 & 0.04 & -0.03 & 0.02 & 0.05 & 0.08 & 0.03 & 0.03 & -0.01 & -0.02 & 0.03 & 0.03 \\
\hline $6000 / 4.0 /-3.0$ & 0.05 & 0.08 & 0.01 & 0.05 & 0.12 & & 0.11 & 0.07 & 0.10 & 0.07 & 0.10 & \\
\hline $5000 / 2.0 /-1.0$ & 0.04 & 0.06 & 0.03 & 0.06 & 0.20 & 0.13 & 0.00 & -0.07 & -0.07 & -0.16 & 0.02 & -0.05 \\
\hline $5000 / 2.0 /-2.0$ & 0.01 & 0.08 & -0.03 & 0.03 & 0.13 & 0.16 & -0.05 & -0.02 & -0.13 & -0.12 & 0.02 & 0.03 \\
\hline $5000 / 2.0 /-3.0$ & -0.02 & 0.06 & -0.12 & -0.06 & 0.23 & 0.29 & 0.10 & 0.18 & 0.06 & 0.10 & 0.07 & \\
\hline
\end{tabular}

Table 3. Comparison of the equivalent widths $w$.

\begin{tabular}{|c|c|c|c|c|c|c|c|c|c|}
\hline \multirow{2}{*}{$T_{\text {eff }}(\mathrm{K})$} & \multirow[t]{2}{*}{$\log g$} & \multirow[t]{2}{*}[\mathrm{Fe}/\mathrm{H}]{} & \multirow[t]{2}{*}[\mathrm{Mg}/\mathrm{Fe}]{} & \multicolumn{2}{|c|}{$w[\mathrm{~m} \AA ̊]$} & \multicolumn{2}{|c|}{$\begin{array}{c}\operatorname{Mg} \text { I } 8736.012 \AA \\
w / w^{*}\end{array}$} & \multicolumn{2}{|c|}{$\Delta A(\mathrm{Mg})$} \\
\hline & & & & Oso & Mer & Oso & Mer & Oso & Mer \\
\hline \multirow[t]{18}{*}{5000} & 1 & +0.50 & 0.0 & 218 & 225 & 1.148 & 1.179 & -0.27 & \\
\hline & & +0.25 & 0.0 & 183 & 205 & 1.164 & 1.226 & -0.25 & \\
\hline & & +0.00 & 0.0 & 154 & 177 & 1.177 & 1.268 & -0.23 & \\
\hline & & -0.25 & 0.1 & 135 & 158 & 1.176 & 1.299 & -0.21 & \\
\hline & & -0.50 & 0.2 & 118 & 135 & 1.171 & 1.302 & -0.18 & -0.11 \\
\hline & & -0.75 & 0.3 & 102 & 111 & 1.170 & 1.272 & -0.15 & -0.10 \\
\hline & & -1.00 & 0.4 & 86 & 89 & 1.164 & 1.210 & -0.13 & -0.08 \\
\hline & & -1.50 & 0.4 & 40 & 33 & 1.109 & 0.927 & -0.06 & +0.03 \\
\hline & & -2.00 & 0.4 & 14 & 9 & 0.985 & 0.667 & +0.01 & +0.18 \\
\hline & 2 & +0.50 & 0.0 & 217 & 211 & 1.077 & 1.170 & -0.11 & \\
\hline & & +0.25 & 0.0 & 180 & 192 & 1.088 & 1.196 & -0.12 & \\
\hline & & +0.00 & 0.0 & 148 & 167 & 1.094 & 1.214 & -0.12 & \\
\hline & & -0.25 & 0.1 & 130 & 151 & 1.090 & 1.230 & -0.10 & \\
\hline & & -0.50 & 0.2 & 112 & 131 & 1.082 & 1.236 & -0.08 & -0.09 \\
\hline & & -0.75 & 0.3 & 97 & 110 & 1.080 & 1.218 & -0.07 & -0.09 \\
\hline & & -1.00 & 0.4 & 82 & 88 & 1.073 & 1.170 & -0.06 & -0.07 \\
\hline & & -1.50 & 0.4 & 39 & 34 & 1.031 & 0.919 & -0.02 & +0.04 \\
\hline & & -2.00 & 0.4 & 14 & 10 & 0.962 & 0.683 & +0.02 & +0.17 \\
\hline
\end{tabular}

Notes. ${ }^{(*)}$ means LTE) and abundance corrections $\Delta A(\mathrm{Mg})$ obtained by us (Oso) with those obtained by Merle et al. (2011, Mer) using the $8736 \AA$ line.

collisionally dominated $\left(C_{i j}>R_{i j}\right)$ even at $\tau_{500 \mathrm{~nm}}=-3$ creating a strong coupling with the ground level of the $\mathrm{Mg}$ II reservoir such that an increase in the collisional rates, leads to an increase in the populations of MgI levels. Abundance corrections become sensitive to CE towards higher $T_{\text {eff }}$, especially for the inter-combination line $4571 \AA$. An increase of the CE rates, makes the population of $3 \mathrm{~s}^{2}{ }^{1} \mathrm{~S}$ increase, while for all the other levels the effect is the opposite making the upper levels of the lines involving $3 \mathrm{~s}^{2}{ }^{1} \mathrm{~S}$ become closer to this level and therefore reducing the non-LTE corrections. Sensitivity to CE increases with metallicity due to the fact that $n_{\mathrm{e}}$ increases, and so does the electron collisional rates $\mathrm{CE}$. $[\mathrm{Mg} / \mathrm{Fe}]$ close to the solar values leads to larger absolute magnesium abundance at higher stellar metallicities leading to stronger lines whose cores form in higher layers in the atmosphere, where the populations become sensitive to $\mathrm{CE}$. The lines with broad wings tend to be sensitive also to $\mathrm{CH}$.

For $T_{\text {eff }} \gtrsim 5000 \mathrm{~K}$ the ground state of $\mathrm{Mg}$ II is thermalised and $\mathrm{Mg} \mathrm{I}$ becomes a minority species. Changes in $\mathrm{CH} 0$ produce the biggest change in the departure coefficients of $\mathrm{Mg}$ I levels. However, this does not necessarily mean that the abundance corrections are more sensitive to $\mathrm{CH} 0$ than to $\mathrm{CH}$ or $\mathrm{CE}$. For example the $8806 \AA$ line has very little sensitivity to $\mathrm{CH} 0$ in atmospheres at these temperatures but the two levels involved in this transition $\left(3 p^{1} \mathrm{P}\right.$ and $\left.3 \mathrm{~d}^{1} \mathrm{D}\right)$ are more sensitive to $\mathrm{CH} 0$ than to $\mathrm{CH}$ or $\mathrm{CE}$ in these atmospheres. $\mathrm{CH} 0$ affects their populations in such a way that the relative populations between those levels are not altered i.e. $\mathrm{CHO}$ do not change the line source function. This leads to similar line profiles and therefore weak sensitivity of $\triangle A(\mathrm{Mg})$ to $\mathrm{CHO} . \mathrm{CH}$ and $\mathrm{CE}$ on the other hand affect the Mg I levels in different ways: the first 4 low-lying Mg I levels have larger populations when those rates are doubled and other levels lower their populations. This changes the relative populations of $3 \mathrm{p}^{1} \mathrm{P}$ with respect to $3 \mathrm{~d}^{1} \mathrm{D}$ making the $8806 \AA$ line source function more sensitivity to $\mathrm{CH}$ and CE. The $8806 \AA$ line opacity on the other hand has similar sensitive to all collisional processes given that the departure coefficient for lower level of this line, $b_{3 \mathrm{p} 1} \mathrm{P}$, increases when any of these collision rates increase.

Figure 3 shows an example where some of the features discussed above can be seen. By increasing CE rate coefficients, the ground level of $\mathrm{Mg}$ I also increases in population while the Mg I excited levels become slightly less populated. Decreasing CE has the opposite effect and the sensitivity of the abundance corrections on CE for the $4571 \AA$ line is quite significant while the $8806 \AA$ line has little sensitivity to the CE rates. When collisional excitation with $\mathrm{CH}$ is modified to $\mathrm{CH} \times 2.0$, the population of the $3 \mathrm{~d}^{1} \mathrm{D}$ level is reduced (blue, thick, dot-dashed line 


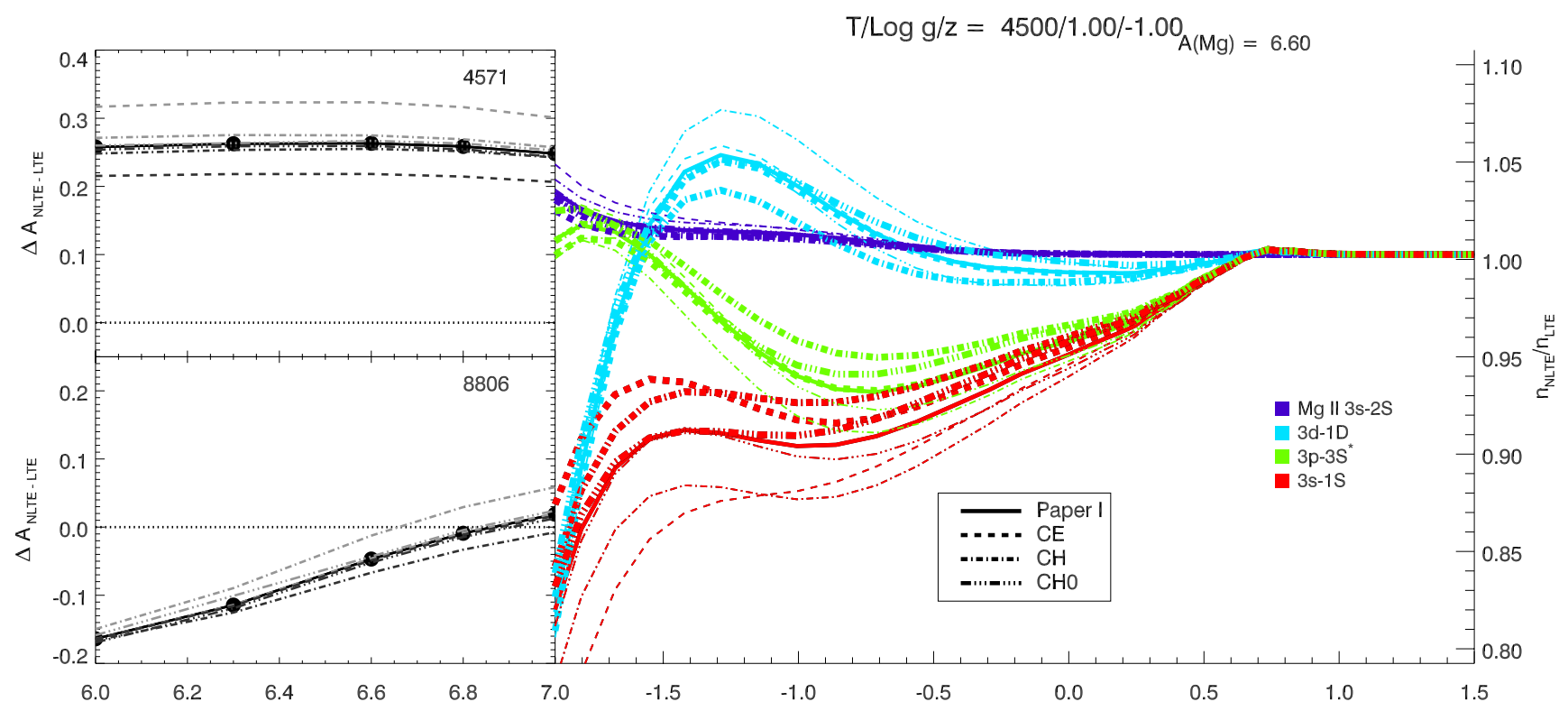

Fig. 3. Sensitivity to collisional data in stellar model $T_{\text {eff }} / \log g /[\mathrm{Fe} / \mathrm{H}]=4500 / 1.0 /-1.0$. Left: abundance corrections $\Delta A(\mathrm{Mg})$ for the $4571 \AA$ (upper $)$ and $8806 \AA$ (lower) lines. The solid lines and dots are $\triangle A(\mathrm{Mg})$ calculated with the model atom F described in Paper I. Broken lines are calculations done with modified collisional rates $C \rightarrow C \times 2.0$ (black) and $C \rightarrow C \times 0.5$ (grey) from the CE (dash), CH (dot-dash) or CH0 (three-dot-dash) rates used in the $\mathrm{F}$ model atom and derived from recent calculations (see text). Right: departure coefficients at $A(\mathrm{Mg})=6.6$ of the levels involved in the $8806 \AA\left(3 \mathrm{~d}^{1} \mathrm{D}-3 \mathrm{p}^{1} \mathrm{P}\right)$ and the $4571\left(3 \mathrm{p}^{3} \mathrm{P}-3 \mathrm{~s}^{1} \mathrm{~S}\right)$ transitions, together with the ground level of $\mathrm{Mg}$ II, as function of optical depth. $3 \mathrm{p}^{1} \mathrm{P}$ has a very similar behaviour to $3 \mathrm{p}^{3} \mathrm{P}$ so it is not shown in the figure to aid visualisation. Solid and broken lines have the same description as in the left figures; thick broken lines are for $C \times 2.0$ and thin broken lines for $C \times 0.5$ rate coefficients.

in the right plot of Fig. 3) compared with the original version of the "F" model atom (blue, solid line). The opposite happens with the $3 \mathrm{p}^{1,3} \mathrm{P}$ levels (green lines). The total effect of $\mathrm{CH} \rightarrow \mathrm{CH} \times 2.0$ on the $8806 \AA$ line (black, dot-dashed line in lower left corner of Fig. 3) is more negative abundance corrections than those obtained with the original $\mathrm{CH}$ rates. Changes in the $\mathrm{CH} 0$ rate coefficients are more uniform among the $\mathrm{Mg}$ I levels, using $\mathrm{CHO} \times 2.0$ leads to an increase of the populations of all the $\mathrm{Mg}$ I levels and $\mathrm{CHO} \times 0.5$ leads to lower Mg I populations (three-dot dashed lines in the right plot). This "uniform shift" of $b_{i}$ with increasing $\mathrm{CH} 0$ observed in Fig. 3 leads to less sensitivity of the nonLTE line profiles to charge exchange collisions for this particular stellar model. As mentioned before, different stars show different effects on different levels so the sensitivity of the non-LTE results to a given collisional process varies across the stellar parameter space.

Table 4 shows the uncertainties associated with the collisional data calculated in Paper I for CE and the data presented in Belyaev et al. (2012), Barklem et al. (2012) for $\mathrm{CH}$ and $\mathrm{CH} 0$. The uncertainties in $w$ associated with these collisional data are $<1 \%(0.005$ dex $)$ for the vast majority of stars and lines. However, for a few stellar models, usually metal-poor, in specific lines and collisional processes, the difference in $w$ due to uncertainties in the collisional data can be as large as $7 \%$ ( 0.03 dex). One exception is change in the $4571 \AA$ line due to CE in the model $T_{\text {eff }} / \log g /[\mathrm{Fe} / \mathrm{H}]=5500 / 4.0 /-4.0$, where the $w$ can be $17 \%(0.07$ dex $)$ smaller if the $\mathrm{CE}$ collisional rates are lowered by $50 \%$. Recall, however, that errors in CE collisional rates are expected to be somewhat smaller, $\sim 20 \%$. The sign in the uncertainty values is related to the direction of change in $w$ relative to the change in the collisional rates. For example, if the $+\mathrm{CH}$ error (i.e., make $\mathrm{CH} \rightarrow \mathrm{CH} \times 2.0$ ) has a negative value then increasing $\mathrm{CH}$ decreases $w$. Likewise, if the $-\mathrm{CH}$ error $(\mathrm{CH} \rightarrow \mathrm{CH} \times 0.5)$ is negative then decreasing $\mathrm{CH}$ increases $w$. These error propagation results reflect the high non-linearity of the non-LTE calculations. For a given stellar atmosphere, a line can have uncertainties in $w$ correlated to a given collisional process and anti-correlated to another, e.g. the star $T_{\text {eff }} / \log g /[\mathrm{Fe} / \mathrm{H}]=5500 / 4.0 /+0.0$ in the $8712 \AA$ line has CE $\& \mathrm{CH}$ anti-correlated errors and at the same time $\mathrm{CH} 0$ correlated errors. Moreover, in some cases, e.g. $\mathrm{CH}$ for the $5172 \AA$ line in the star 4500/3.0/-4.0, increasing and decreasing $\mathrm{CH}$ rates, both lead to smaller abundance corrections ${ }^{5}$.

\subsection{Differential effects}

Applications of abundance studies are usually interested in using or comparing abundances from stars of different types, i.e. their relative abundances. For example, it is of great interest to trace abundance changes with stellar evolutionary status among populations, e.g. globular clusters, to understand stellar processes. Another example, is the study of differences in elemental abundances in various parts of the Galaxy (thin and thick disks and halo), and to identify new components. The detection of new substructure will require high precision, and in order to identify stars of different types to a common population (sometimes called chemical tagging) or have the best statistics we must be able to accurately compare abundances from stars of different types (e.g. Lindegren \& Feltzing 2013).

An interesting question is how non-LTE affects the differences in abundances between stars with different stellar parameters, compared with LTE modelling. In Fig. 4, each panel shows the difference in non-LTE abundance

$\Delta[\mathrm{Mg} / \mathrm{Fe}]_{\mathrm{NLTE}}=[\mathrm{Mg} / \mathrm{Fe}]_{A_{\mathrm{NLTE}}}-[\mathrm{Mg} / \mathrm{Fe}]_{B_{\mathrm{NLTE}}}$

5 By checking the line profiles of the $5172 \AA$ line, the $\mathrm{CH} \times 2$ predicted line has wider wings and a weaker core than the $\mathrm{CH} \times 0.5$ predicted line, leading to similar equivalent widths. 


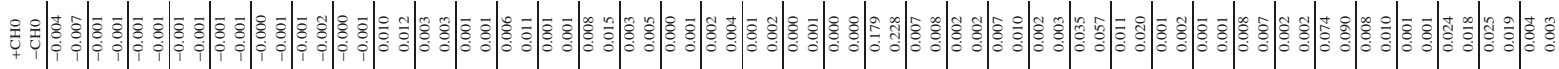

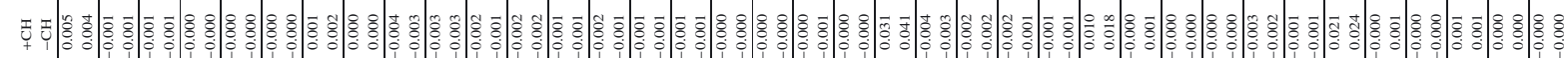

U

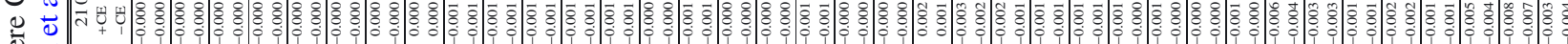

$\stackrel{\square}{\circ}$

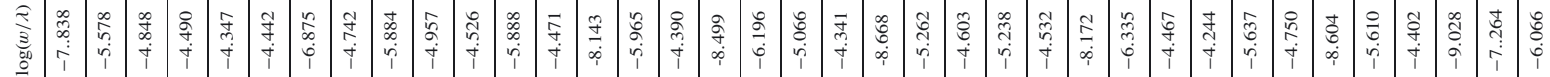

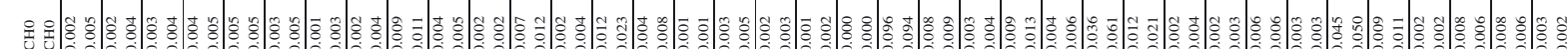

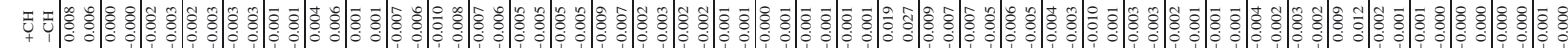

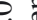

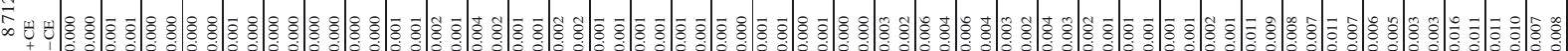

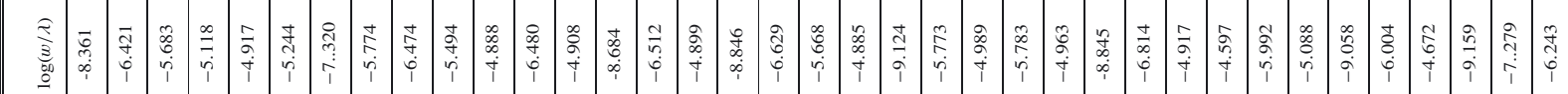

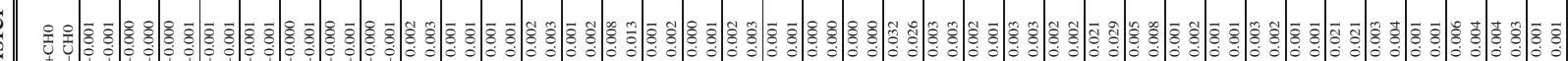

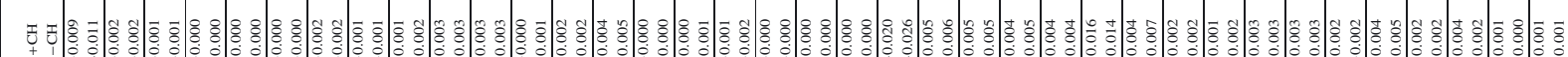

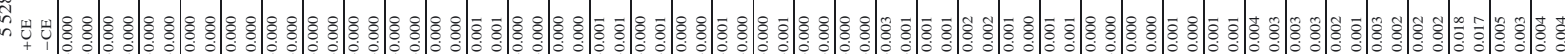

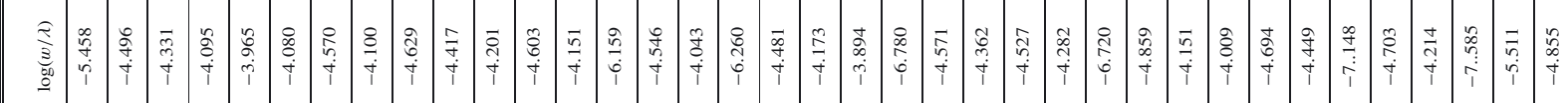

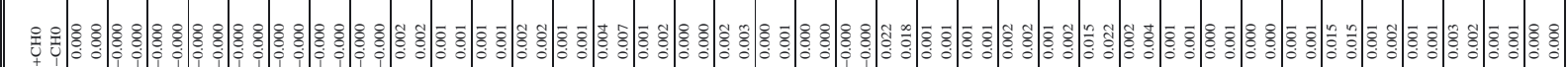

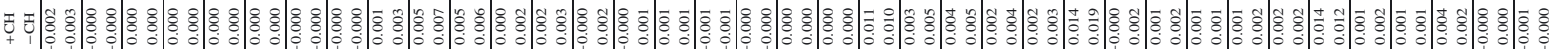

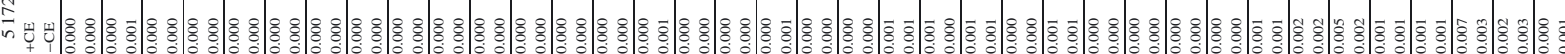

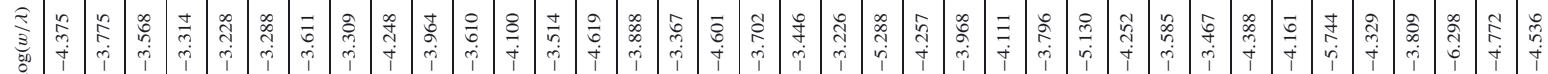

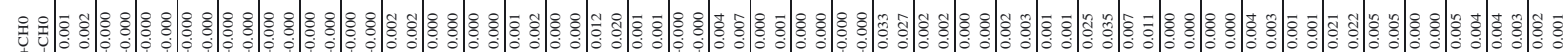

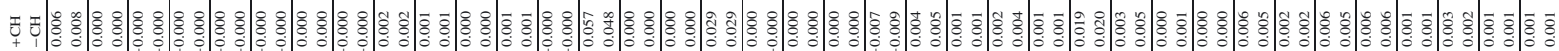

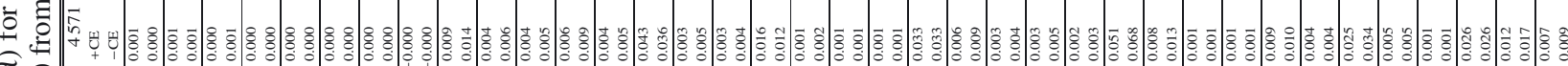

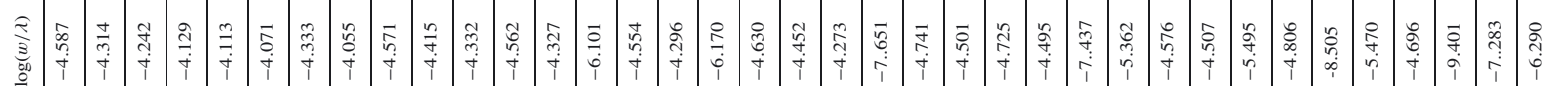

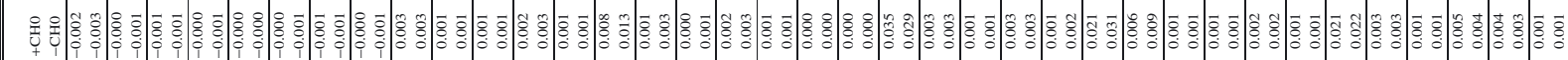

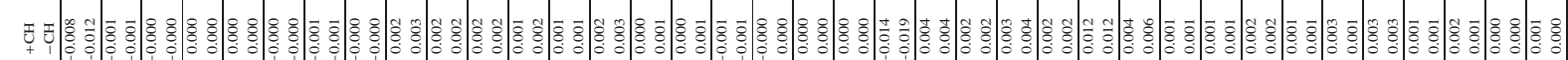

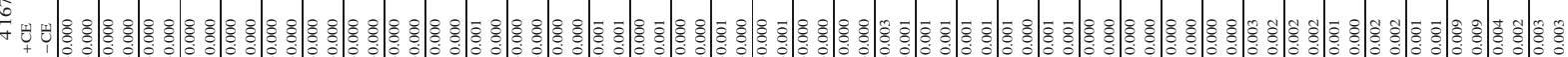

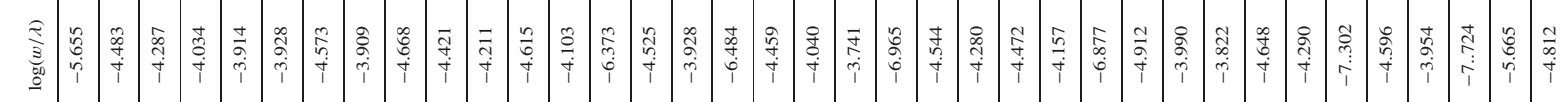

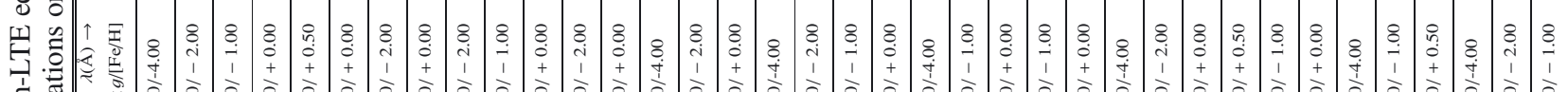

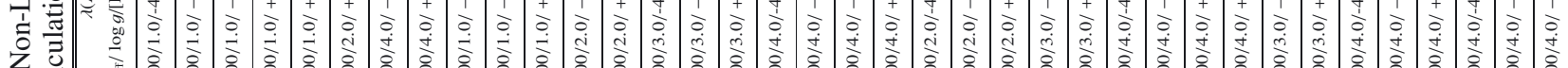


Y. Osorio and P. S. Barklem: Mg Line formation in late-type stellar atmospheres
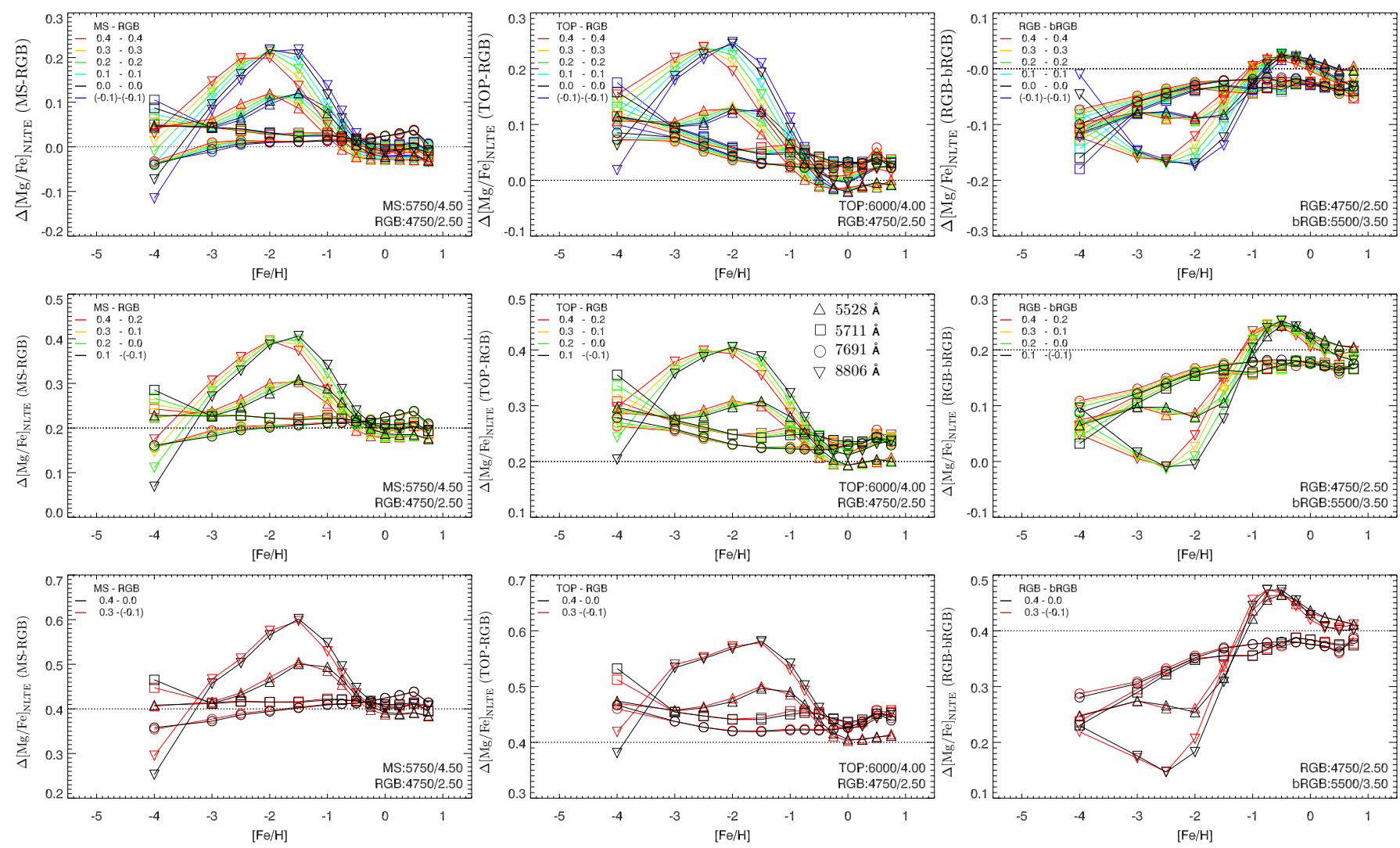

Fig. 4. Difference in non-LTE Mg abundance $\Delta[\mathrm{Mg} / \mathrm{Fe}]_{\mathrm{NLTE}}$ versus stellar metallicity for a fixed LTE difference $\Delta[\mathrm{Mg} / \mathrm{Fe}]_{\mathrm{LTE}}(=0,0.2$ and 0.4 in the first, second and third row respectively), between two stars with $T_{\text {eff }} / \log g$ parameters shown at the bottom right of each plot. The LTE difference $\Delta[\mathrm{Mg} / \mathrm{Fe}]_{\mathrm{LTE}}$ is represented by the dotted line. Colours indicate the LTE abundance $[\mathrm{Mg} / \mathrm{Fe}]_{\mathrm{LTE}}$ for each star shown at the top-left of each plot. Four different $\mathrm{Mg}$ I lines were used: 5711(口), 7691( $\bigcirc), 5528(\triangle)$ and $8806(\nabla) \AA$. Examples of individual stars at various evolutionary stages were selected: main sequence (MS), turn off point (TOP), red giant branch (RGB) and base of the RGB (bRGB).

for two stars A and B with the same metallicity and difference in $\mathrm{Mg}$ LTE abundance $\Delta[\mathrm{Mg} / \mathrm{Fe}]_{\mathrm{LTE}}$ (represented by the dotted line). We call $\Delta[\mathrm{Mg} / \mathrm{Fe}]_{\mathrm{NLTE}}$ the differential abundance correction. The first row in Fig. 4 shows the difference in $\Delta[\mathrm{Mg} / \mathrm{Fe}]_{\mathrm{NLTE}}$ when the two stars have the same $[\mathrm{Mg} / \mathrm{Fe}]_{\mathrm{LTE}}$ abundance; different values of $[\mathrm{Mg} / \mathrm{Fe}]_{\mathrm{LTE}}$ are represented in different colours. Examples of individual stars at various evolutionary stages were selected: main sequence (MS), turn off point (TOP), red giant branch (RGB) and base of the RGB (bRGB). The most noticeable feature is the increase of non-LTE differential abundance correction with decreasing metallicity. Red giant stars have their largest abundance corrections for the $8806 \AA$ line around $[\mathrm{Fe} / \mathrm{H}]=-2$, and as a consequence differences are stronger at these values of $[\mathrm{Fe} / \mathrm{H}]$. For example if a metal-poor RGB star with $T_{\text {eff }} / \log g /[\mathrm{Fe} / \mathrm{H}]=4750 / 2.5 /-2.0$ shows $[\mathrm{Mg} / \mathrm{Fe}]_{\mathrm{LTE}}=$ 0.1 dex and a bRGB star with $T_{\text {eff }} / \log g /[\mathrm{Fe} / \mathrm{H}]=5500 / 3.5 /-2.0$ shows $[\mathrm{Mg} / \mathrm{Fe}]_{\mathrm{LTE}}=-0.1 \mathrm{dex}$, the LTE abundance difference is 0.2 dex (black lines in the right, middle plot in Fig. 4) but the non-LTE abundance difference obtained using the $8806 \AA(\nabla)$ line is 0.0 dex and using the $5528 \AA(\Delta)$ line is 0.1 dex.

Comparison of the magnitude of evolutionary trends of $\mathrm{Mg}$ abundances seen in globular clusters (e.g. Fig. 6 in Korn et al. 2007) with the magnitudes of non-LTE effects seen, underlines the necessity to include accurate non-LTE corrections in order to study changes in atmospheric abundances with evolutionary status (see also Gruyters et al. 2013, 2014). Figure 5 shows the $\mathrm{Mg}$ abundance one would derive in LTE as function of $T_{\text {eff }}$ for various values of $\log g$ for a constant non-LTE abundance. That is, it shows the predicted trend of abundance with evolutionary status that one would find if non-LTE effects are neglected and if the atmospheric $\mathrm{Mg}$ abundance were the same in the compared stars.

\section{Conclusions}

We obtained departure coefficients and equivalent widths for selected diagnostic $\mathrm{Mg}$ lines, which were presented and described. The departure coefficient data, while the equivalent width data and (full Table 1) are available at the CDS. The non-LTE abundance corrections are usually small but not negligible. Giants tend to have negative corrections while dwarfs have in general positive corrections. Abundance corrections increase at low metallicities. These results are in general agreement with earlier studies; however, the magnitude of the corrections is often different (usually smaller) due to our improved collision data. We have demonstrated the necessity to account for non-LTE for precise differential analysis between giants and dwarfs.

The non linearity of the non-LTE problem leads to a complicated error propagation and Table 4 can be used as guide to uncertainties in non-LTE abundances due to collisional data in different regions of the stellar parameter space. In general uncertainties due to collisional data lead to uncertainties in derived abundances of $<0.01 \mathrm{dex}(2 \%)$, although in some cases can be as large as 0.03 dex (7\%). As these errors are less than or of the same order as typical corrections, we expect that we can use these results to extract $\mathrm{Mg}$ abundances from high quality spectra more reliably than from classical LTE analysis. 

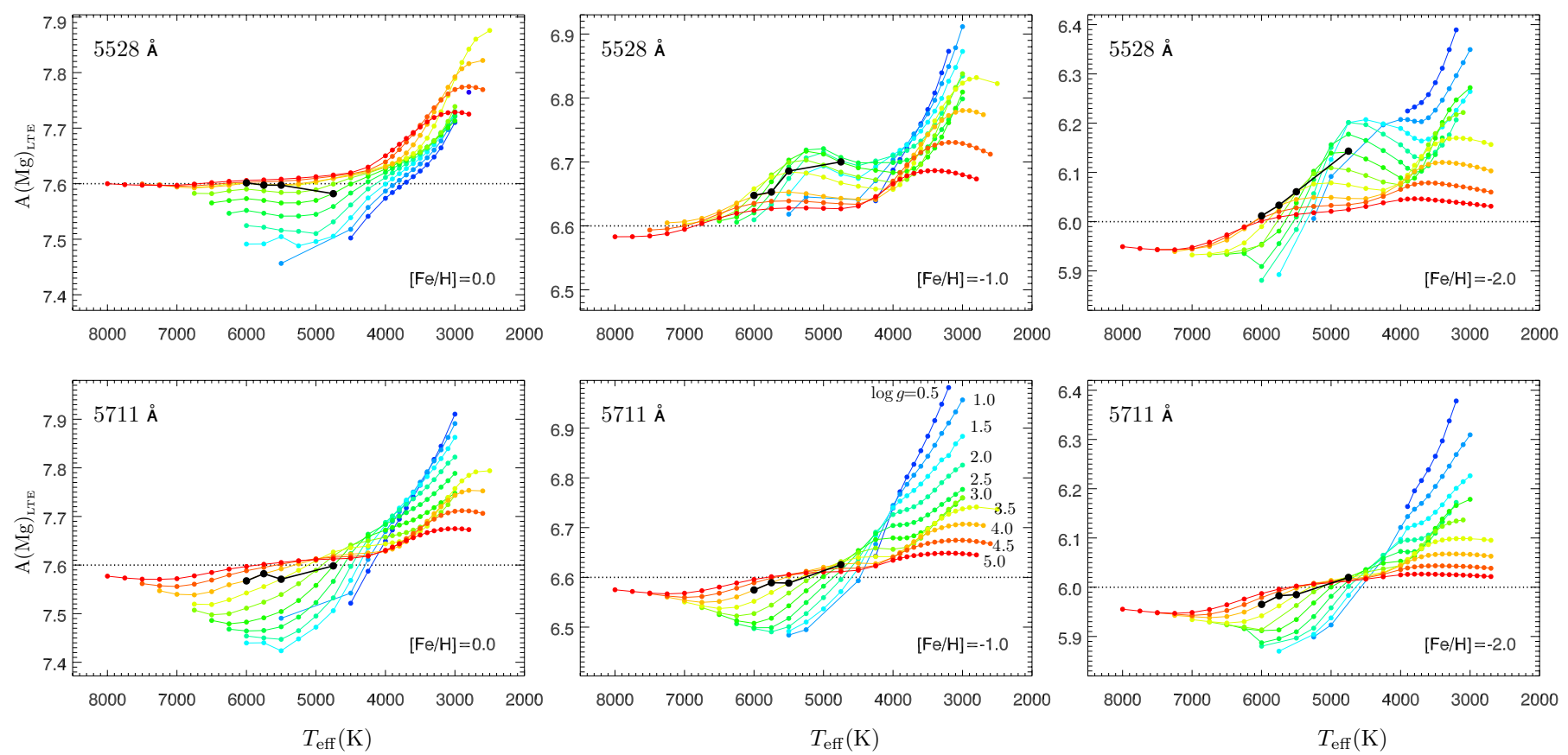

Fig. 5. For a fixed Mg non-LTE abundance (dotted line), the Mg abundance that would be derived in LTE shown as function of $T_{\text {eff }}$ based on the 5528 (top) and 5711 (bottom) $\AA$ lines. Colour lines connect model atmospheres with the same $\log g\left(\mathrm{~cm} / \mathrm{s}^{2}\right.$ ). $\log g$ varies from 0.5 (blue) to 5.0 (red) dex in steps of 0.5 dex. The black points correspond to the RGB, bRGB, SGB and TOP models used in Fig. 4.

Acknowledgements. The computations were performed on resources provided by SNIC through Uppsala Multidisciplinary Center for Advanced Computational Science (UPPMAX) under Project SNIC 2014/1-220. This work was supported by the Royal Swedish Academy of Sciences, the Wenner-Gren Foundation, Göran Gustafssons Stiftelse and the Swedish Research Council. P.S.B. is a Royal Swedish Academy of Sciences Research Fellow supported by a grant from the Knut and Alice Wallenberg Foundation. P.S.B. was also supported by the project grant "The New Milky" from the Knut and Alice Wallenberg foundation.

\section{References}

Athay, R. G., \& House, L. L. 1962, ApJ, 135, 500

Barklem, P. S., Anstee, S. D., \& O’Mara, B. J. 1998, PASA, 15, 336

Barklem, P. S., Belyaev, A. K., Spielfiedel, A., Guitou, M., \& Feautrier, N. 2012, A\&A, 541, A80

Belyaev, A. K., Barklem, P. S., Spielfiedel, A., et al. 2012, Phys. Rev. A, 85, 032704

Carlsson, M. 1986, Uppsala Astronomical Observatory Reports, 33

Carlsson, M. 1992, in Cool Stars, Stellar Systems, and the Sun, ed. M. S. Giampapa \& J. A. Bookbinder, Conf. Ser., 26, 499

Carlsson, M., Rutten, R. J., \& Shchukina, N. G. 1992, A\&A, 253, 567

Carlsson, M., Rutten, R. J., Bruls, J. H. M. J., \& Shchukina, N. G. 1994, A\&A, 288,860

Chang, E. S., Avrett, E. H., Noyes, R. W., Loeser, R., \& Mauas, P. J. 1991, ApJ, 379, L79

Christlieb, N., Bessell, M. S., Beers, T. C., et al. 2002, Nature, 419, 904 Dimitrijevic, M. S., \& Sahal-Brechot, S. 1996, A\&AS, 117, 127

Fabbian, D., Asplund, M., Carlsson, M., \& Kiselman, D. 2006, A\&A, 458, 899

Frebel, A., Aoki, W., Christlieb, N., et al. 2005, Nature, 434, 871
Fuhrmann, K., Pfeiffer, M., Frank, C., Reetz, J., \& Gehren, T. 1997, A\&A, 323, 909

Gruyters, P., Korn, A. J., Richard, O., et al. 2013, A\&A, 555, A31

Gruyters, P., Nordlander, T., \& Korn, A. J. 2014, A\&A, 567, A72

Guitou, M., Belyaev, A. K., Spielfiedel, A., Feautrier, N., \& Barklem, P. S. 2012,

J. Phys.: Conf. Ser., 397, 2053

Gustafsson, B., Edvardsson, B., Eriksson, K., et al. 2008, A\&A, 486, 951

Heiter, U., \& Eriksson, K. 2006, A\&A, 452, 1039

Kaulakys, B. P. 1986, J. Exper. Theor. Phys., 91, 391

Korn, A. J., Grundahl, F., Richard, O., et al. 2007, ApJ, 671, 402

Langangen, Ø., \& Carlsson, M. 2009, ApJ, 696, 1892

Lind, K., Asplund, M., \& Barklem, P. S. 2009, A\&A, 503, 541

Lind, K., Asplund, M., Barklem, P. S., \& Belyaev, A. K. 2011, A\&A, 528, A103

Lind, K., Bergemann, M., \& Asplund, M. 2012, MNRAS, 427, 50

Lindegren, L., \& Feltzing, S. 2013, A\&A, 553, A94

Mashonkina, L. 2010, in EAS Pub. Ser. 43, eds. R. Monier, B. Smalley, G. Wahlgren, \& P. Stee, 189

Mashonkina, L. 2013, A\&A, 550, A28

Mauas, P. J., Avrett, E. H., \& Loeser, R. 1988, ApJ, 330, 1008

Merle, T., Thévenin, F., Pichon, B., \& Bigot, L. 2011, MNRAS, 418, 863

Osorio, Y., Barklem, P. S., Lind, K., et al. 2015, A\&A, 579, A53 (Paper I)

Przybilla, N., Butler, K., Becker, S. R., \& Kudritzki, R. P. 2001, A\&A, 369, 1009

Ralchenko, Y., Kramida, A., Reader, J., \& NIST ASD Team 2010, NIST Atomic Spectra Database (version 5.0), [Online]. Available: http://physics. nist.gov/asd [2013, March 7]

Seaton, M. J. 1962, in Atomic and Molecular Processes, ed. D. R. Bates, 375

Shimanskaya, N. N., Mashonkina, L. I., \& Sakhibullin, N. A. 2000, Astron. Rep., 44,530

Sundqvist, J. O., Ryde, N., Harper, G. M., Kruger, A., \& Richter, M. J. 2008, A\&A, 486, 985

Uitenbroek, H., \& Noyes, R. W. 1996, Cool stars; stellar systems; and the sun 9, ASP Conf. Ser., 109, 723

Valenti, J. A., \& Piskunov, N. 1996, A\&AS, 118, 595 